\title{
Regulation of Human Mesenchymal Stem Cell Osteogenesis by Specific Surface Density of Fibronectin: a Gradient Study
}

\author{
Ana B. Faia-Torres, ${ }^{\dagger, \dagger, \S}$ Tolga Goren, ${ }^{\S}$ Teemu O. Ihalainen, ${ }^{\perp, \square}$ Stefanie Guimond-Lischer, ${ }^{\#}$
} Mirren Charnley, ${ }_{\S}^{, \Psi}, \Psi$ Markus Rottmar, ${ }^{\#} \triangle$ Katharina Maniura-Weber, $^{\#}$ Nicholas D. Spencer, ${ }^{\S}$ Rui L. Reis, ${ }^{\dagger, \ddagger}$ Marcus Textor, ${ }^{\S}$ and Nuno M. Neves $*, \dagger, \ddagger$

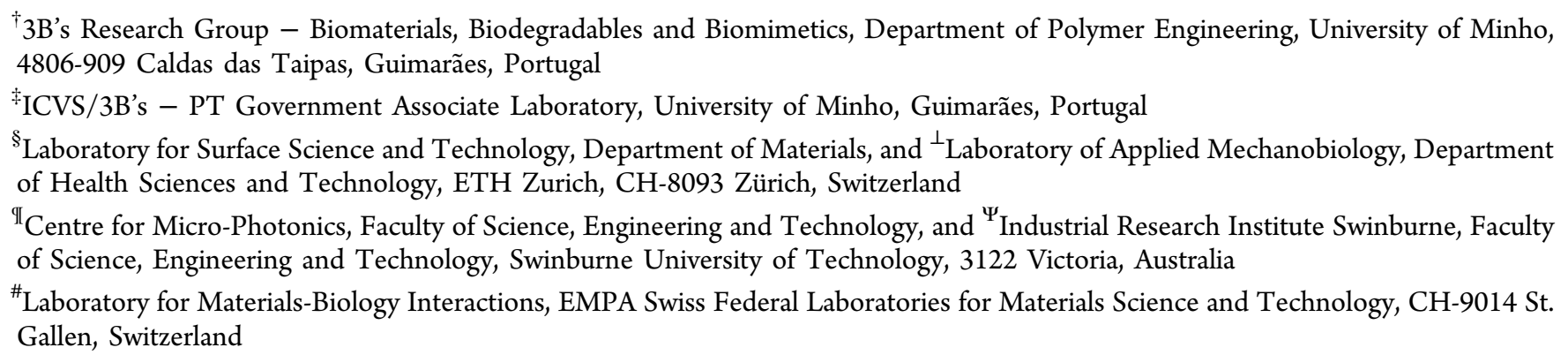

\section{Supporting Information}

ABSTRACT: The success of synthetic bone implants requires good interface between the material and the host tissue. To study the biological relevance of fibronectin (FN) density on the osteogenic commitment of human bone marrow mesenchymal stem cells (hBMMSCs), human FN was adsorbed in a linear density gradient on the surface of PCL. The evolution of the osteogenic markers alkaline phosphatase and collagen 1 alpha 1 was monitored by immunohistochemistry, and the cytoskeletal organization and the cell-derived FN were assessed. The functional analysis of the gradient revealed that the

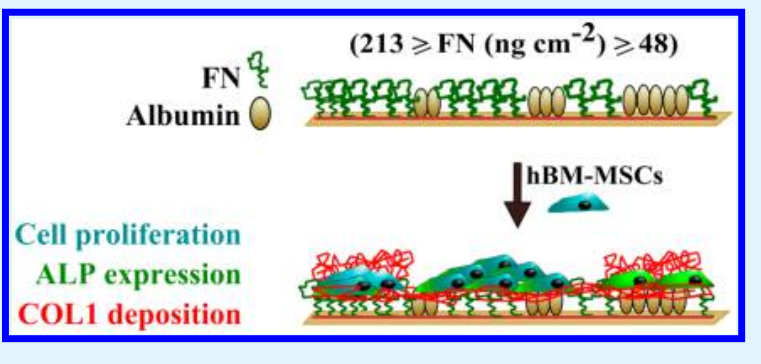
lower FN-density elicited stronger osteogenic expression and higher cytoskeleton spreading, hallmarks of the stem cell commitment to the osteoblastic lineage. The identification of the optimal FN density regime for the osteogenic commitment of hBM-MSCs presents a simple and versatile strategy to significantly enhance the surface properties of polycaprolactone as a paradigm for other synthetic polymers intended for bone-related applications.

KEYWORDS: biomaterials, gradient, fibronectin, mesenchymal stem cells, osteogenesis

\section{INTRODUCTION}

Imparting biomimetic characteristics to the implant surface of synthetic materials is essential to reinforce the interaction between implant and host tissue. ${ }^{1}$ Synthetic scaffold materials are typically characterized by uniform and reproducible properties, but they lack the bioactivity and positive host tissue response associated with extracellular matrix (ECM)-derived scaffolds. ${ }^{2,3}$ Weak tissue-implant integration often leads to low mechanical stability, inflammation and, ultimately, rejection of the implants, compromising the efficacy of therapies. ${ }^{4}$ Coating of synthetic implants with natural adhesion proteins of the ECM may contribute to the enhancement of the interactions between the implant and the host tissues, which ultimately strengthens the tissue-implant interface. The main protein of bone is collagen type 1 (COL1). ${ }^{5}$ However, the COL1 polymerization was shown to require the presence of $\mathrm{FN}^{6,7}$ This requirement may implicate $\mathrm{FN}$ in the formation of matrix defects such as those seen in osteogenesis imperfecta, which is caused by qualitative or quantitative deficiencies in COL1, and results in bones prone to fracture. ${ }^{8}$ Furthermore, there is evidence that $\mathrm{FN}$ is synthesized and deposited during tissue repair ${ }^{9}$ where early recruitment and commitment of osteoblast precursors, such as mesenchymal stem cells (MSCs), occurs. Human bone marrow-MSCs (hBM-MSCs) can differentiate into a variety of adult cell types, such as osteoblasts, chondrocytes or adipocytes. ${ }^{10}$ Hence, the trigger and support of the osteogenic differentiation of hBM-MSCs by the scaffoldmaterial at the lesion site, is critical for the success of the bone tissue regeneration. Recent advances have shown that, along with soluble factors, biophysical, biomechanical, and cell adhesion protein cues can also be strong regulators of stemcell fate. ${ }^{11-13}$ The presence of FN is not bone ECM-specific, so it was hypothesized that the distinctive role of $\mathrm{FN}$ in the osteogenic commitment of hBM-MSCs could be related to a

Received: October 9, 2014

Accepted: December 16, 2014

Published: December 16, 2014 
specific optimal density of this protein in the ECM-microenvironment. Surface gradients allow for the screening of specific physicochemical properties, and have been successfully applied in a number of cell-biology studies. ${ }^{13-16}$ This approach allows the identification of the gradient region that elicits the optimum cell response of interest, while keeping the other experimental conditions consistent. The advantages of this method include determining the limits by which each surface characteristic is effective, minimizing cell-culture variations, decreasing the cost of consumables, and reducing the probability of methodological errors due to the large number of samples that would otherwise be necessary to test similar hypotheses. The later advantage is particularly important for the study of stem cell populations, which may be difficult to obtain in large numbers and are significantly affected in their stemness by the in vitro expansion step. ${ }^{17}$

In this study were engineered $10 \mathrm{~mm}$-long gradients, consisting of a linear surface-density arrangement of human plasma-isolated FN adsorbed on polycaprolactone (PCL). The surface was further passivated using bovine serum albumin, which is known to inhibit MSC adhesion. ${ }^{18}$ This system allowed for the systematical study of the effect of FN density, varying from densely packed to sparse molecule distributions, on the biological performance of hBM-MSCs. The bioperformance of each surface density area was assessed by the analysis of the cell adhesion and proliferation, production of endogenously cell-derived FN (endoFN), and expression of the osteoblastic markers alkaline phosphatase (ALP) and collagen type 1 alpha 1 (COL1A1). To the best of our knowledge, this is the first systematic study reporting the density-specific influence of adsorbed human FN on the osteogenic commitment of hBMMSCs.

\section{MATERIALS AND METHODS}

Surface Preparation. Silicon wafers with a native oxide coating (Si-Mat Silicon Materials, Germany), $1 \mathrm{~cm} \times 1 \mathrm{~cm}$, were sonicated twice for $10 \mathrm{~min}$ in toluene, then twice for $10 \mathrm{~min}$ in 2-propanol, and finally exposed to oxygen plasma for 2 min (PDC-32G instrument, Harrick, Ossining, NY, USA). The thickness of the silicon oxide layer was then measured using variable-angle spectroscopic ellipsometry (VASE; described below). The polycaprolactone (PCL; TONE, Union Carbide Chemicals and Plastics Division; NJ, USA) solution was prepared at a concentration of $3 \mathrm{~g} / \mathrm{L}$ in tetrahydrofuran (SigmaAldrich). Ultrathin polymeric films were obtained by spin-coating (Fairchild Technologies, 1001, Germany) of the silicon wafers at room temperature for $70 \mathrm{~s}$ at $4000 \mathrm{rpm}$. The substrates were then annealed overnight at $90{ }^{\circ} \mathrm{C}$ and submitted to air-plasma (a distinct PDC-32G instrument, Harrick, Ossining, NY, USA) for $34 \mathrm{~s}$, at $60 \mathrm{~W}$ and 0.1 mbar, to proceed to the sterilization of the sample.

Polycaprolactone (PCL) Surface Characterization. Static contact-angle measurements were performed with $4 \mu \mathrm{L}$ water drops, at room temperature, with a NRL contact-angle goniometer (model 100-00-230, Ramé Hart Inc.; NJ, USA). Six measurements were carried out for each sample.

Thickness measurements were performed before and after PCL spin-coating, as well as after air-plasma treatment, using a variableangle spectroscopic ellipsometer (VASE) (M-2000F, L.O.T. Oriel $\mathrm{GmbH}$, Germany) equipped with a focusing probe, at room temperature, with an incidence angle of $70^{\circ}$ on several spots of each wafer, over the spectral range of $370-994 \mathrm{~nm}$. The resulting measurements were fitted with the WVASE32 analysis software to a multilayer model for a silicon substrate, silicon oxide layer and a polymer adlayer with the optical properties of a Cauchy film. ${ }^{19}$

Isolation of Fibronectin (FN) from Human Plasma. FN was isolated from human plasma (Zürcher Blutspendedienst SRK) following a previously published gelatin-sepharose chromatographic method. ${ }^{20}$ Briefly, after adding $2 \mathrm{mM}$ phenylmethylsulfonyl fluoride and $10 \mathrm{mM}$ EDTA to the fresh human plasma, the plasma was centrifuged at $15000 \mathrm{~g}$ for $40 \mathrm{~min}$. Plasma was passed to the gelatin Sepharose 4B column (Pharmacia) and the flow-through was directly passed to the Sepharose 4B column (Sigma-Aldrich). The gelatin column was then washed with $2 \mathrm{mM}$ phenylmethylsulfonyl fluoride and $10 \mathrm{mM}$ EDTA in PBS (Fluka Chemicals, Buchs). Before elution, the gelatin column was washed with $1 \mathrm{M} \mathrm{NaCl}, 1 \mathrm{M}$ urea, and finally FN was eluted from the column with $6 \mathrm{M}$ urea. Isolated $\mathrm{FN}$ was stored at $-20{ }^{\circ} \mathrm{C}$ in $6 \mathrm{M}$ urea. Before use for surface coating, the isolated FN was dialyzed against PBS (for $16 \mathrm{~h}$ at room temperature). Dialyzed FN was further stored at $-20^{\circ} \mathrm{C}$ for not more than 3 months.

Alexa-633 FN Labeling. FN ( $2 \mathrm{mg} / \mathrm{mL}$ in PBS) was labeled using fluorescent marker Alexa-633-NHS (Invitrogen) according to the manufacturer's instructions. Briefly, 1:10 volume of $1 \mathrm{M}$ bicarbonate buffer ( $\mathrm{pH}$ 8.0) was added to the FN solution to increase the reactivity of the NHS ester. Alexa-633-NHS was dissolved in DMSO $(10 \mathrm{mg} /$ $\mathrm{mL}$ ) and added to the protein solution to reach a final concentration of $1 \mathrm{mg} / \mathrm{mL}$. The solution was incubated in the dark at room temperature for $2 \mathrm{~h}$ and then dialyzed against PBS. Finally, the labeled FN was stored at $-20^{\circ} \mathrm{C}$ in the dark.

Production of Gradients Using the Labeled-FN. Transmission interference adsorption sensor (TInAS) measurements ${ }^{21}$ were used to determine the adsorption kinetics of FN on PCL-coated substrates. TInAS sensors (which possess a silicon oxide surface coating) were spin-coated with PCL using the same procedure as described above in Surface Preparation. The sensors were equilibrated in a $10 \mathrm{mM}$ solution of phosphate-buffered saline (PBS) (Fluka Chemicals, Buchs), and then a solution of $0.04 \mathrm{mg} / \mathrm{mL}$ of FN in PBS was introduced at a constant flow rate of $20 \mu \mathrm{L} / \mathrm{min}$. This flow rate was determined in previous studies to correspond well to gradient dipping conditions, preventing depletion of the buffer without artificially accelerating the adsorption. The adsorption curve was characterized by rapid initial adsorption of the protein onto the surface, which slowed down as the proteins rearranged themselves on the surface until no further adsorption took place. The results showed that for a concentration of $0.04 \mathrm{mg} / \mathrm{mL}$ of $\mathrm{FN}$, the saturation level was obtained after a controlled immersion time of $60 \mathrm{~min}$. The sensors were then rinsed with PBS without variation in the measured optical thickness. The FN adsorption curve was translated into a dipping program designed to yield a linear gradient. This program was followed by a computer-controlled motor arm (OWIS, Staufen, Germany) to immerse the PCL-coated substrates into a beaker with the Alexa 633-FN solution at a controlled rate. Following FN-gradient production, the substrates assigned for characterization were immediately rinsed in PBS and ultrapure water, and dried under a nitrogen steam. The gradients assigned for cell culture were, instead, rinsed in PBS and incubated for $1 \mathrm{~h}$ at room temperature in a solution of $4 \%$ bovine serum albumin (BSA; Sigma, A6003), after which the samples were rinsed again twice and stored at $4{ }^{\circ} \mathrm{C}$ in $\mathrm{PBS}$ until cell seeding.

Characterization of the FN Gradient. The linearity of the produced FN gradient was determined by VASE measurements, taken $2 \mathrm{~mm}$ apart along the length of the gradient, before and after protein adsorption, using VASE equipped with a focusing probe with an incidence angle of $70^{\circ}$, at room temperature over the spectral range of 370-994 nm. The resulting measurements were fitted with the WVASE32 analysis software to a multilayer model for a silicon substrate, a silicon oxide layer (of the measured thickness prior to PCL treatment), and PCL and FN adlayers with the optical properties of a Cauchy film. ${ }^{19}$ Microscopy imaging was used to qualitatively confirm the presence of the labeled FN gradient. Quantitative analysis of the intensity of the fluorescent signal was determined by the histogram algorithm in ImageJ software ${ }^{22}$ (Wayne Rasband, National Institutes of Health, USA) before and following the cell-culture. All measurements were plotted as mean \pm standard error.

Isolation of Human Bone Marrow Mesenchymal Stem Cells (hBM-MSCs). The isolation of primary human bone marrow mesenchymal stromal cells (hBM-MSCs) from femur-derived bone marrow samples was accomplished after the informed consent of three 


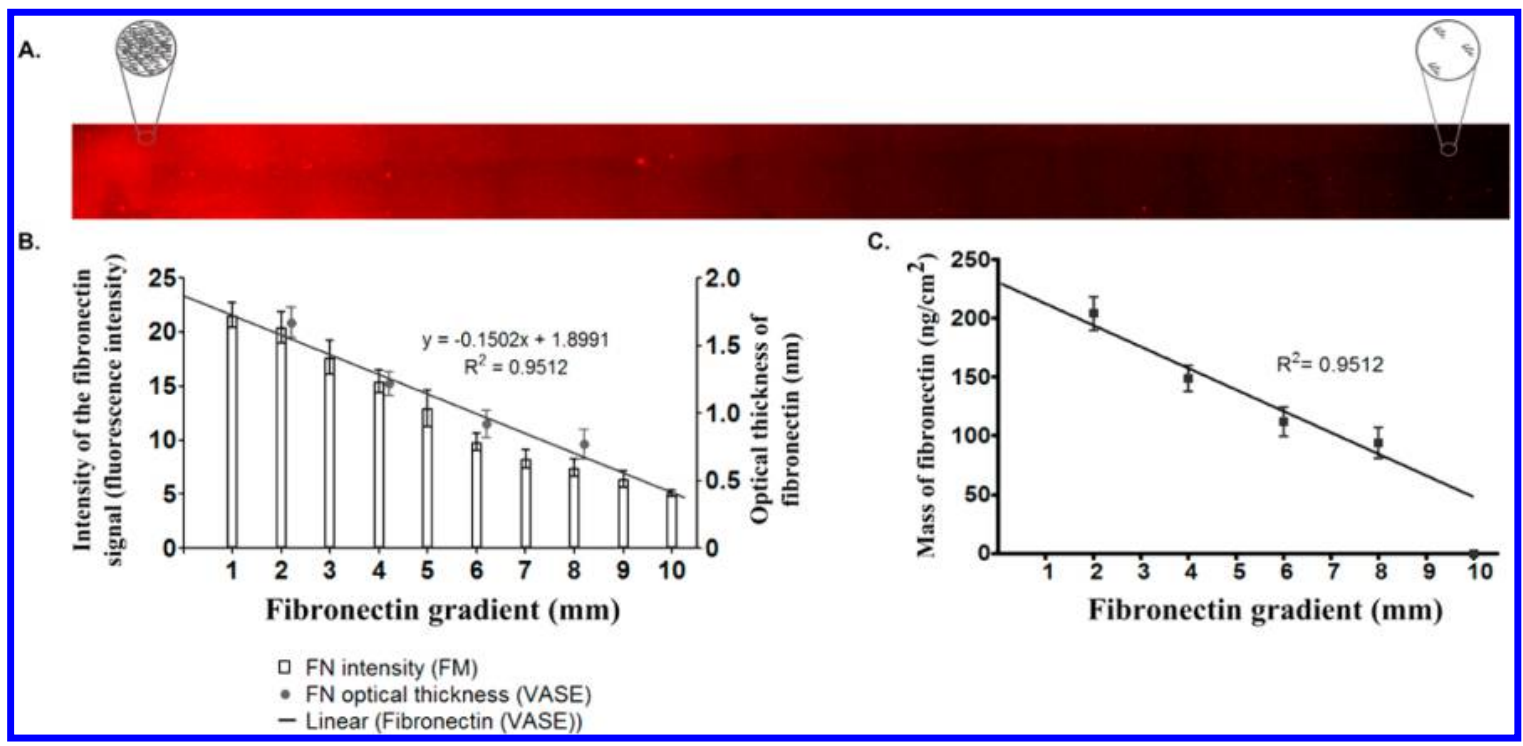

Figure 1. (A) Acquisition by fluorescence microscopy (FM) of the fluorescence signal of fibronectin (FN) along the $10 \mathrm{~mm}$ surface of polycaprolactone (scale bar $=1 \mathrm{~mm}$ ), confirming the confluent to sparse distribution of FN, as indicated by the inset drawings. (B) Quantification of the optical thickness of FN measured by variable-angle spectroscopic ellipsometry, and of the fluorescence intensity of FN measured by FM. (C) Mass of FN along the gradient, calculated from the optical thickness measurements obtained by transmission interference adsorption sensing, using the de Feijter equation. ${ }^{24}$

independent donors (67-82 years old). The protocol was approved by the ethics board of the Kantonspital St. Gallen, Switzerland (EKSG 08/14). As described before, ${ }^{23}$ bone-marrow samples were incubated in isolation medium (25 mM HEPES (Fluka, 54459), $128.5 \mathrm{mM} \mathrm{NaCl}$ (Fluka, 71380), $5.4 \mathrm{mM} \mathrm{KCl}$ (Fluka, 60130), $5.5 \mathrm{mM} \mathrm{d}(+)$-glucose (Sigma, G7528), $51.8 \mathrm{mM} \mathrm{d}(+)$-saccharose (Sigma, 84097), 0.1\% bovine serum albumin (Sigma, A6003)) overnight at $4{ }^{\circ} \mathrm{C}$. The protocol proceeded with the centrifugation of the bone marrow at $110 \mathrm{~g}$ for $15 \mathrm{~min}$ at $4{ }^{\circ} \mathrm{C}$ to remove fat tissue in the supernatant. The cell solution was ressuspended in isolation medium, filtered by a 200 $\mu \mathrm{m}$ polyethylene terephthalate (PET) filter, and centrifuged. After the supernatant had been discarded by aspiration, the cell pellet was resuspended in basal growth medium ( $\alpha$-MEM supplemented with $10 \%$ fetal bovine serum (FBS; Lonza), $1 \%$ penicillin-streptomycinneomycin antibiotic mixture $(100 \times)$ (PSN; Gibco), $1 \mathrm{ng} / \mathrm{mL}$ fibroblast growth factor 2 (FGF-2; Sigma, F0291)). The cell number in solution was determined and the cells seeded at a density of $1 \times 10^{7}$ cells in a T75 culture flask in basal growth medium. After $24 \mathrm{~h}$, the nonadherent cells were removed and the medium was changed twice weekly from then on. Cells were subcultured at subconfluence and further seeded at passage one or two.

Seeding and Culture of hBM-MSCs. hBM-MSCs were seeded as a concentrated drop at 5000 cells $/ \mathrm{cm}^{2}$ in basal growth medium on $\mathrm{FN}$ gradient samples (in circular wells of nonadherent tissue culture polystyrene of internal diameter of $21.40 \mathrm{~mm}$ ) and on cell-adherent tissue culture polystyrene well-plates (TCPS). The cell attachment occurred for $4 \mathrm{~h}$, after which fresh medium was added to complete 1.5 $\mathrm{mL}$ of medium per well. Twenty- $4 \mathrm{~h}$ later, the basal growth medium was aspired and substituted by standard osteogenic induction medium ( $\alpha$-MEM supplemented with 10\% FBS, 1\% PSN, $50 \mathrm{mM}$ ascorbate-2phosphate (Sigma, A8960), $2 \mathrm{mM} \beta$-glycerophosphate (Sigma, G9891), $10 \mathrm{nM} \mathrm{1,25-dihydroxyvitamine} \mathrm{D3} \mathrm{(Sigma,} \mathrm{D-1530),} \mathrm{and} 10$ $\mathrm{nM}$ dexamethasone (Sigma, D-4902)), both in the FN gradient samples and in the TCPS.

Immunofluorescence. At each time point, cells on the control material TCPS and on the FN gradient samples were removed from the incubator, gently washed twice in prewarmed PBS and fixed and permeabilised with $4 \%$ paraformaldehyde $/ 0.2 \%$ Triton X-100 for 8 min. The samples were then thoroughly washed in PBS without glucose (Gibco, 18912-014) and placed in 1\% fetal calf serum/5\% goat serum for $30 \mathrm{~min}$ at room temperature, to block nonspecific binding sites. Following the incubation period the samples were rinsed (twice) in PBS without glucose and incubated with the antibodies. The primary antibodies, recommended for detection of antigens of human origin, were used as follows: alkaline phosphatase (ALP) (1:1000, Developmental Hybridoma Bank, B4-78), collagen type 1 alpha 1 (1:200, Santa Cruz Biotecnology, 8784) and fibronectin (1:100, Santa Cruz Biotechnology, 6953), in 3\% BSA in PBS for $1 \mathrm{~h}$ at room temperature (RT). The samples were then rinsed (twice) in PBS without glucose and cell nuclei were fluorescently stained by 4'-6diamidino-2-phenylindole (DAPI; 1:1000, Sigma-Aldrich, D9542) in $3 \%$ BSA in PBS, for $1 \mathrm{~h}$ at RT, combined with the secondary antibodies: Alexa Fluor 546 goat antimouse IgG (1:400, Molecular Probes, A11030) and Alexa Fluor 488 goat antirabbit IgG (1:400, Molecular Probes, A11034) for ALP and COL1A1 reactivity; and donkey antigoat antibody Cy3 conjugate (1:400, Merck Millipore, AP180C) for human fibronectin detection.

Independent Detection of Adsorbed FN and Endogenously Cell-Derived FN. The FN gradient was fabricated using Alexa-633 conjugated plasma-FN (Figure 1A) which allowed direct and quantitative measurement of the FN content adsorbed on the PCLcoated substrate, on the first, seventh and 14th day/time points (Figure 2). Additionally, to assess the endogenously cell-derived FN

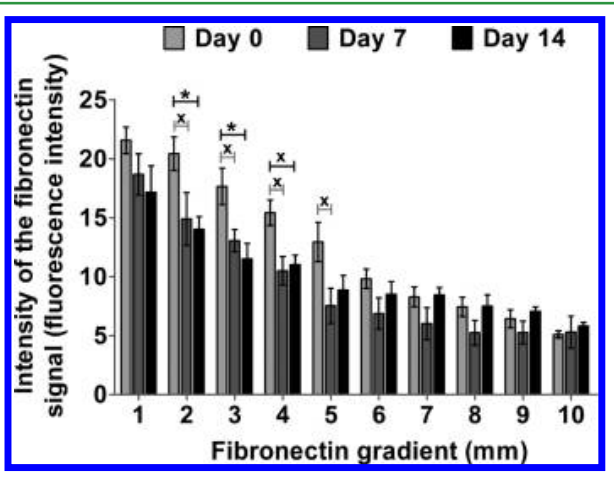

Figure 2. Variation in the fibronectin (FN) density on polycaprolactone-coated wafers prior to cell seeding (day 0 ) and at the 7 th and 14th days of cell culture, as a function of the intensity of the FN fluorescence signal. The statistical significance of the measurements was $p(*)<0.01$ and $p(\mathrm{x})<0.05$. 
(endoFN) on the samples it was necessary to proceed with the immunolabeling of the (total) FN content, by a primary anti-FN antibody combined with a secondary antibody (above detailed). Unexpectedly, the acquisition of the fluorescence signal coming from the total FN content was not able to detect the originally adsorbed FN. Example images from the 14th day are shown here: originally adsorbed Alexa-633 FN (A), and antibody antihuman FN (B). When merging both channels, no superposition of red and green is evident (C), showing that the originally adsorbed FN, independently confirmed to still be present via Alexa-633 detection, was not observable by this method. This may result from the comparably higher concentration of fluorochromes bounding to and brightening the multiple-layers of the endoFN (compared to the sparse-tomonolayer-like distribution of the originally adsorbed FN), which ultimately allowed for the independent observation of the endoFN on the samples. If one wished to detect the originally deposited FN through this channel, one would have to increase the intensity of the laser, superexposing the fibers of the endoFN on top of the originally adsorbed FN.

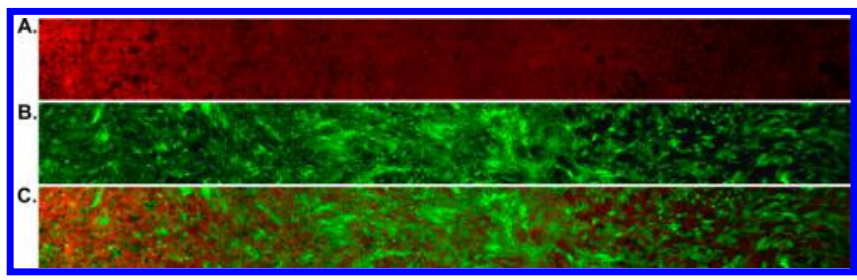

Fluorescence Microscopy and Quantification of the Intensity of the Fluorescent Signal. Microscopy was performed by an automated fluorescence microscope (Zeiss Axiovert 200M), using the DAPI, Alexa Fluor 488, Alexa Fluor 546/Cy3 and the Alexa 633 emission filter settings. A 20x objective was used to image and capture by automated acquisition the $10 \mathrm{~mm}$ length of the gradient. Four millimeters in the perpendicular axis were also captured, and the set of images stitched, as a mosaic, by the AxioVision 4.8.1 software available with the microscope. To allow for the comparison of the intensity of the fluorescence signal of the ALP, COL1A1, and endoFN, we kept the microscope settings constant after the determination of the optimal conditions to capture the brightest sample of each biomarker set. The images were then analyzed by ImageJ software, ${ }^{22}$ which quantified the variation of the fluorescence intensity within each gradient sample (per $\mathrm{mm}^{2}$ ) and TCP controls. For that, the fluorescence signal corresponding to the immunodetection of ALP, COL1A1 and FN was converted into the gray scale and the mean intensity of the gray values per $\mathrm{mm}^{2}$ was calculated by the histogram algorithm. The background signal was also measured and, if different from zero, subtracted from the original measurement to generate the accurate intensity of expression of each biomarker. Finally, to determine the intensity of the fluorescent signal of ALP, COL1A1 and FN normalized to the cell count, the cell nuclei per square millimeter was also quantified. For that, the mean average of the nuclei \pm standard deviation was calculated, using the scale bar as a reference, and a band size filter applied to exclude any artifacts, such as dust particles. Moreover, the nuclei image was segmented by the watershed algorithm to prevent overlapping nuclei to be counted as single cells. The cytoskeletal area along the length of the FN gradient and on TCPS was directly calculated by the space occupied by the F-actin per $\mathrm{mm}^{2}$ of single cells in the image. However, when the agglomerate of cells did not allow for the direct measurement, the total cytoskeletal area was divided by the number of nuclei in the respective space. All normalized results are presented as the mean \pm standard error of the mean value of the replicates.

Statistical Analysis. Statistical analysis was performed using GraphPad (version 5.01 for Windows, San Diego, California). Twoway ANOVA test followed by Bonferroni posttest were performed to analyze the effect of substrate (TCPS or FN gradients) and culture time (in days) on the (1) cell density and distribution, (2) normalized expression of the osteogenic markers ALP and COL1A1, (3) normalized expression of endoFN and (4) cytoskeleton area. The significance level of the threshold was set at 0.05 . Thus, $p$ (probability) values lower than 0.05 were considered to describe statistical significance.

\section{RESULTS AND DISCUSSION}

Linearly Varying FN-density Gradient on PCL, and the FN Rearrangement Throughout the Study. To study the osteogenic commitment of hBM-MSCs on variable FN surfacedensity, we engineered a linear FN density-gradient onto PCL. The kinetics of FN adsorption onto PCL were measured using a previously published in situ transmission interference adsorption sensing (TInAS) technique. ${ }^{21}$ The resulting adsorption curve was inverted and translated into a dipping program. Substrates were immersed in FN solution by a computer-controlled arm following the generated dipping program, yielding a linear density gradient over the length of the substrates. The production of the FN gradient at the surface of the PCL-coated wafers was confirmed by fluorescence microscopy and variable-angle spectroscopic ellipsometry (VASE) (Figure 1). The positive correlation between the fluorescence intensity of FN and VASE measurements is demonstrated in the results of Figure 1B. The VASE data additionally shows that the FN adsorption promotes a maximum variation of $1.5 \mathrm{~nm}$ in the coating thickness. Considering the poor contrast offered by proteins, such a difference in surface morphology cannot be detected by SEM, although it poses no problem for optical techniques. The specific adsorbed mass of FN on the PCL substrates (calculation detailed in the Supporting Information) linearly varied from a densely packed arrangement to a sparse FNmolecule distribution, from $\sim 213$ to $48 \mathrm{ng} / \mathrm{cm}^{2}$ along the 10 mm-long gradient (Figure 1C).

Cells receive inputs from the ECM and the soluble factors, and process them to secrete and remodel the matrix. ${ }^{25,26}$ The rearrangement of the adsorbed matrix proteins at the material interface is important in guiding cell activity. In fact, the inhibition of the $\mathrm{FN}$-material reorganization, such as that supported by covalent bonds, has been shown to prompt proteolytic activity to degrade FN in order to introduce microscale modifications that direct cell function. ${ }^{18}$ In our adsorbed-FN gradient platform, the desorption of FN was only statistically significant at the higher-density half of the gradient (positions $2-5 \mathrm{~mm}$; Figure 2). This protein rearrangement on the surface could be due to the fact that the newly produced proteins (such as COL1A1 and endoFN) could not be accommodated within the more closely packed FN layer, forcing the FN exchange at the surface. In contrast, the apparently intact FN density adsorbed on the lower-density half of the gradient (positions $6-10 \mathrm{~mm}$ ) can be the result of the increasingly high density of bovine serum albumin (BSA) backfilling the FN gradient from the first to the tenth $\mathrm{mm}$. It is known that smaller-molecular-weight proteins and more highly concentrated proteins are typically displaced from the surface by larger molecular weight proteins (a competitive exchange commonly referred as the "Vroman effect"). ${ }^{28}$ For that reason, in the lower FN density region, the BSA (a low-molecularweight protein ${ }^{27}$ ) was more prone to desorption than the highmolecular-weight $\mathrm{FN}^{29}$ justifying the apparently intact FN arrangement on those regions. Overall, despite the FN surface rearrangement, a gradient in the density range of the originally adsorbed FN was still evident throughout the 14 days of cell culture (Figure 2). 
Effectiveness of the FN Gradient and TCPS on the Proliferation and Osteogenic Commitment of hBMMSCs. At day 1, the cell density on the TCPS was not statistically different from the cell density at the various positions of the FN gradient (Figure 3B). From day 1 to day 7 ,

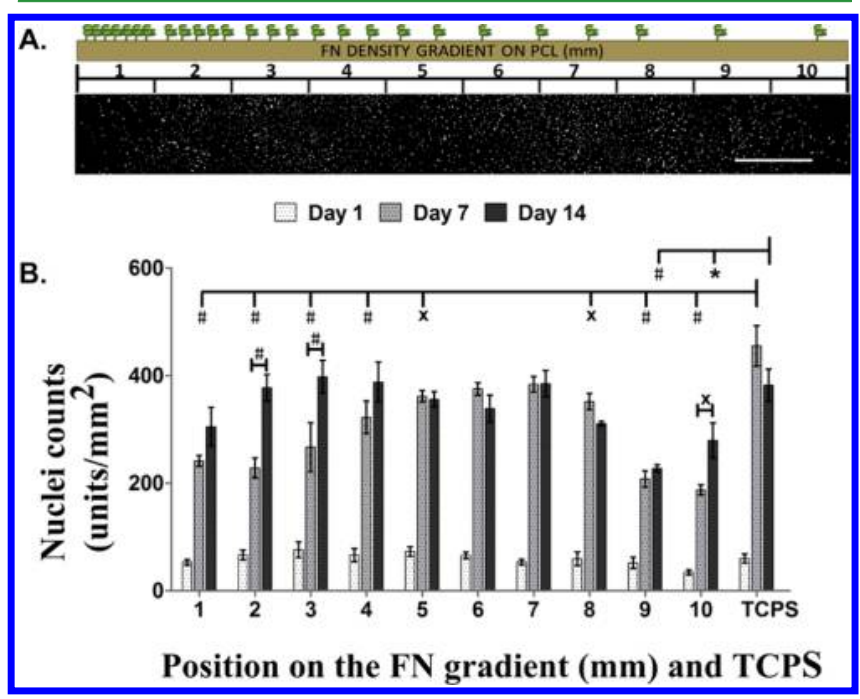

Figure 3. (A) Fluorescence microscopy image representative of cell nuclei distribution at day 1 of cell culture on the fibronectin (FN) gradients (scale bar $=1 \mathrm{~mm}$ ). (B) Quantification of cell nuclei on FN gradients and tissue culture polysteryne (TCPS) at day 1,7 , and 14 . The statistical significance of the measurements was $p(\mathrm{x})<0.05, p(*)$ $<0.01$ and $p(\#)<0.001$.

however, the increase in the cell numbers across the FN gradient ranged between 3.5- and 7-fold (Figure 3B). The results indicate that the cell proliferation was affected by the FN-density on the surface, possibly due to the differential exposure of the cryptic binding-sites in $\mathrm{FN}$, critical for stimulating increasing cell proliferation. ${ }^{30,31}$ At day 7 , the cell density highlighted the division of the gradient into two functional groups: FN surface densities inducing higher cell numbers (regions from 4 to $8 \mathrm{~mm}$ ), and FN surface densities inducing lower cell numbers (regions from 1 to 3 and 9 to 10 $\mathrm{mm}$ ). Since the extremities of the FN gradient correspond to extreme confluent and sparse coverage of adsorbed FN, the lack of a statistically significant difference in the cell density was unexpected. However, the similarity may be explained by the molecular arrangement of FN in the gradient. There are several binding sites in the FN molecule, which include both proteinand cell-binding sites. ${ }^{31}$ Among them, there are at least four known FN-FN binding sites. We believe that at the higher density end of the FN gradient, the proximity between neighboring FN molecules adsorbed on the surface may lead to FN-FN binding, thereby hiding cell-binding sites. On the other hand, the lower density end of the FN gradient presents a direct correlation between lower coverage and number of cellbinding sites. Given that hBM-MSCs are adherent cells, the eventual reduction of the number of cell-binding sites available may contribute to the low cell numbers in those areas. At the same time point, TCPS supported higher cell density than all positions of the FN gradient $(0.001<p<0.05)$, except for positions 6 and $7 \mathrm{~mm}(p>0.05)$. From day 7 to day 14, the cell number increase occurred only on positions 2,3 , and $10 \mathrm{~mm}$ in the FN gradient (Figure 3B). However, even at those positions, the cell-number expansion was substantially smaller than that seen from day 1 to day 7 .

The reduced cell proliferation is usually associated with an increase in the differentiation commitment of BM-MSCs. ${ }^{32}$ Indeed, confirming this, already at day 7 the analysis showed that the extremities of the gradient more effectively influenced the osteoblastic induction of the stem-cell population. At those positions, a greater cell spreading and cuboidal phenotype were detected, particularly evident at the 10th $\mathrm{mm}$ in the gradient $(p$ $<0.001$ ) (see Figure S1 in the Supporting Information). Both features are linked with the commitment to the osteoblastic lineage. ${ }^{33}$ In the same time point (day 7), on the extremities of the gradient the osteoblastic induction was further characterized by augmented 1) ALP expression and 2) synthesis of COL1A1, both normalized to cell number (Figures $4 B$ and $5 B$ ).

The synthesis (and presecretion stage) of COL1A1, which was determined by the fluorescence signal surrounding the cell nucleus at day 7 , was also seen on TCPS (Figure 5A). In the same time point, the normalized COL1A1 and ALP signals were markedly lower on the control material than at the extremities of the FN gradient ( $\sim 4.5$ - to 6.5 -fold and $\sim 5$-fold, respectively, $0.001<p<0.01)$. Remaining positions on the gradient (i.e., 2 to $8 \mathrm{~mm}$ ) showed comparable performance to that of TCPS at supporting the ALP and COL1A1 expression $(p>0.05)$. The data implies a differential regulation of the osteogenic commitment of hBM-MSCs on the FN-gradient. Moreover, density-specific FN environments have the ability to trigger stronger osteogenic commitment than the control material, TCPS. Increased hBM-MSC commitment to the osteoblast lineage in the presence of $\mathrm{FN}$ has been previously reported, although that study was not quantitative with respect to FN density. ${ }^{34}$ In fact, the general limited information on the characteristics of the protein-coatings, such as the final proteindensity at the surface, hinders the cross-integration of the conclusions of different studies, and may explain apparently conflicting results. ${ }^{35}$

Along the same lines (i.e., lower proliferation relating to cell commitment to differentiation), the normalized ALP expression on the TCPS registered a significant increase from day 7 to day 14 ( $\sim 6.5$ fold), in accordance to previously published data. ${ }^{35}$ The effectiveness of the TCPS matched that of positions 7 and $9 \mathrm{~mm}$ in the FN gradient $(p>0.05)$ and surpassed the effectiveness of all other positions $(0.01<p<0.05)$ with exception of position $10 \mathrm{~mm}$. In fact, the normalized ALP expression doubled on the 10th $\mathrm{mm}$ on the gradient, from day 7 to day $14(p<0.001)$ (Figure $4 \mathrm{~B})$; as a result, it was $\sim 40 \%$ higher than the control, at day 14. Moreover, also at day 14, the normalized expression of COL1A1 increased $\sim 2$ to 5 times on the FN gradients $(0.001<p<0.01)$, with the greatest increases observed at the first, ninth and tenth millimeter (Figure 5). The normalized COL1A1 expression on TCPS was 5 to 6 times lower than that at the extremities of the gradient, and $\sim 3.5$ to 4 times smaller than the normalized expression at the remaining positions (i.e., $2-8 \mathrm{~mm}$ ), confirming the positive influence of FN on the COL1 polymerization ${ }^{6}$ even in suboptimal FN densities. Moreover, along with the extracellular fibril arrangement of COL1A1 on the FN gradient and TCPS at day 14, the strong intracellular signal of COL1A1 seen at day 7 persisted on the FN gradient surface, but not on the TCPS (Figure 5A). This observation seems to indicate that the continuous synthesis of collagen, the major protein of bone, is induced by environments where $\mathrm{FN}$ is steadily available, given the 


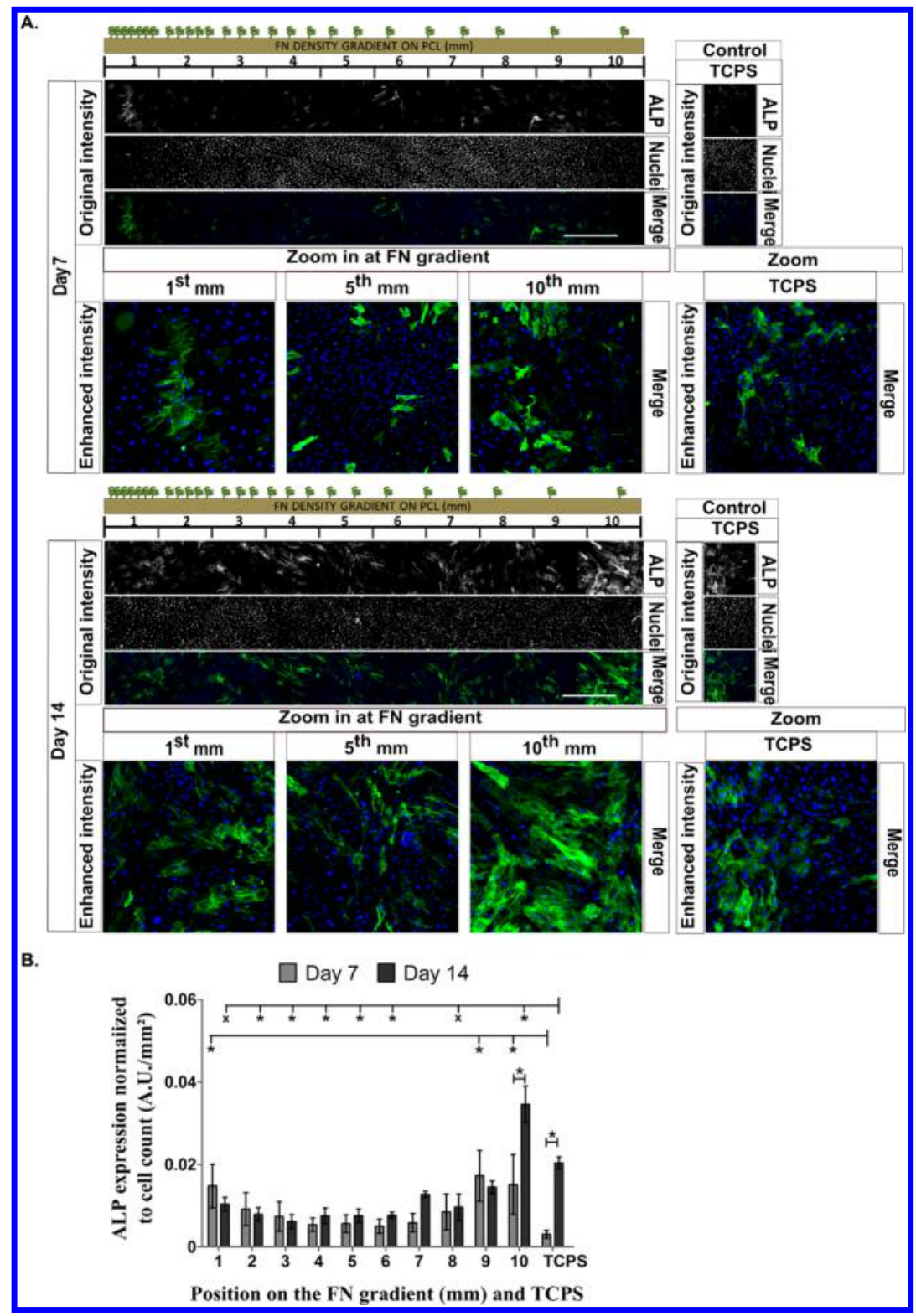

Figure 4. Expression of alkaline phosphatase (ALP) on fibronectin (FN) gradients and tissue culture polystyrene (TCPS), at days 7 and 14 . (A) Immunodetection of ALP by fluorescence microscopy (scale bar $=1 \mathrm{~mm}$ ). All square images represent $1 \times 1 \mathrm{~mm}$. (B) Quantification of ALP expression normalized to cell count. The statistical significance of the measurements was $p(*)<0.01$ and $p(\mathrm{x})<0.05$.

regulatory role of $\mathrm{FN}$ on the deposition and retention of COL1 in the matrix. ${ }^{7,36}$

Interestingly, at the last time point, the expression of COL1A1 in the matrix assumed cottony-fibrillar morphology (Figure 5A) similar to the endogenously cell-derived FN (endoFN) on the FN gradient (Figure 6A). It has been proposed that the assembly of FN and collagen share common mechanistic elements, which in association with the requirement of FN for collagen fibril assembly ${ }^{6}$ can influence in determining the final morphology of the COL1A1 fibrils. The morphological similarity between the COL1A1 on our FN gradient (but not in the TCPS control) and reported FN around osteoblasts during osteogenesis in vivo ${ }^{37}$ at the same time point (14 days), is compelling. The similar fibrillar arrangement, here referred as in vivo-like, of the FN and COL1A1 matrices deposited by the differentiating hBM-MSC populations (following the introduction of the specific adsorbed FN-density cue) may add considerable value to constructs for bone-related applications. In fact, is has been shown that how hMSCs proactively pull on newly assembled FN ECM fibrils affects and (up)regulates hMSC osteogenesis. ${ }^{38}$ However, the relationship between COL1A1 and FN seems to go beyond the FN's influence on the COL1 expression and fibrillogenesis. In this study, the presence of COL1A1 in the ECM at day 14, in comparison to its predominant intracellular expression at day 7 , correlated with a significant drop in the endoFN expression on the gradient (Figure 6B). The observation seems to indicate a control of the endoFN by the COL1A1 in the matrix, favoring the osteogenic process. Furthermore, the differential deposition of endoFN further highlighted the importance of total lower FN content (i.e., endoFN plus plasma-FN) for the maximization of the osteogenic commitment, characterized by the simultaneous increase of ALP and COL1A1 expression in the ECM at position $10 \mathrm{~mm}$. In comparison, higher plasma-FN showed the ability to significantly influence the COL1A1 but not the ALP activity. The interaction between plasma-derived 


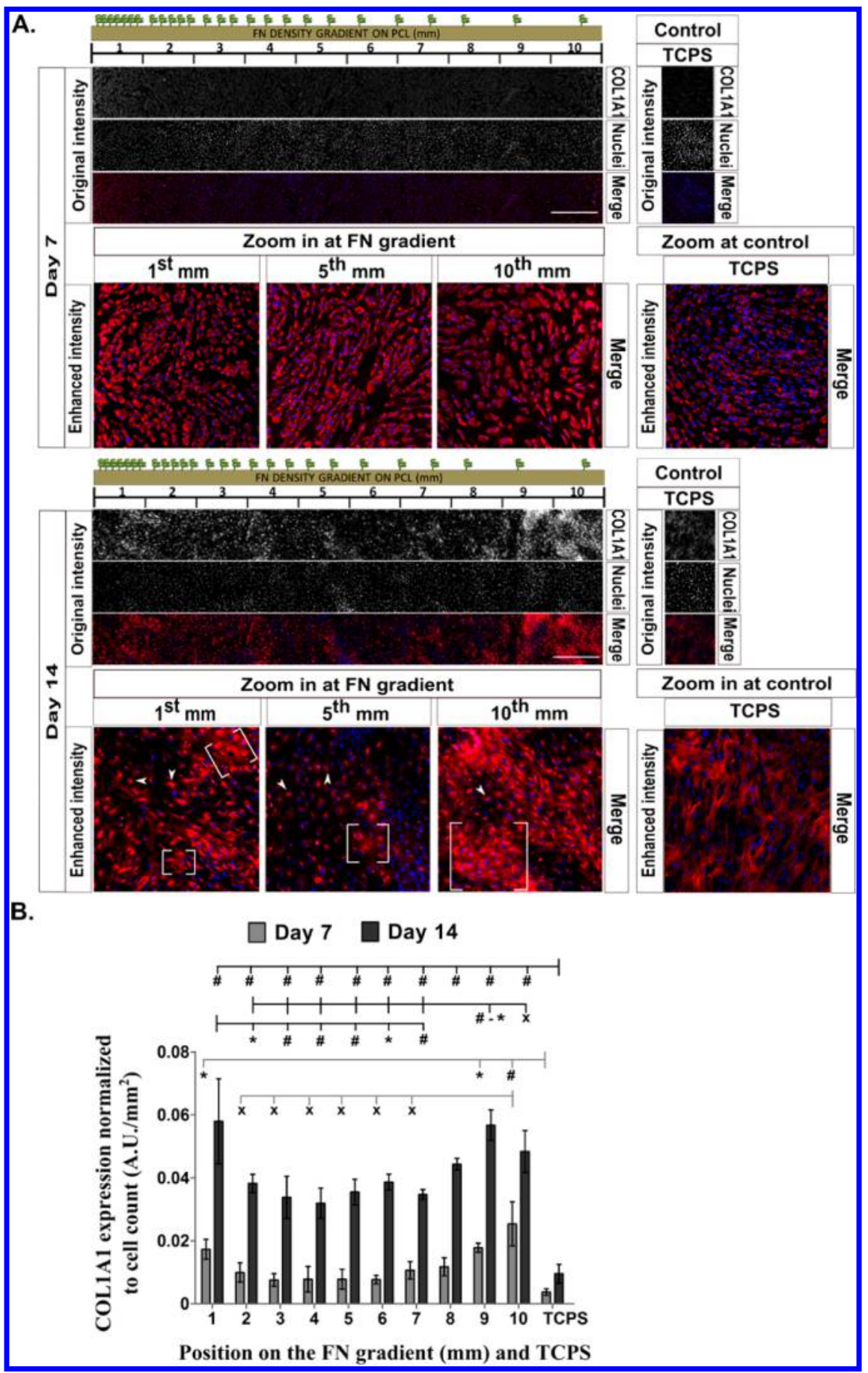

Figure 5. Expression of collagen type 1 alpha 1 (COL1A) on fibronectin (FN) gradients and tissue culture polystyrene (TCPS), at days 7 and 14 . A) Immunodetection of COL1A1 by fluorescence microscopy (scale bar $=1 \mathrm{~mm}$ ). All square images are $1 \times 1 \mathrm{~mm}$. Representative intracellular COL1A1 is indicated by arrows, whereas fibrils of extracellular COL1Alare indicated by brackets. (B) Quantification of the COL1A1 expression, normalized to cell number. The statistical significance of the measurements was $p(\#)<0.001, p(*)<0.01$, and $p(\mathrm{x})<0.05$.

FN (as that composing the FN gradient) and collagen assembly in bone was previously noticed in vivo. ${ }^{39}$ Moreover, in accordance to our findings, the authors observed no effect of plasma-FN over the modulation of the osteoblastic function.

Together with the differentiation mechanism elicited by the osteogenic induction medium, specific (low) fibronectin density significantly affected and maximized the osteoblastic lineage commitment of the stem cell population. The influence of the FN in the osteogenic commitment may result from the induced changes in cell shape (see Figure S1 in the Supporting
Information), as the cytoskeleton is known to play a significant role in mechano-transduction and MSC differentiation. ${ }^{38,40}$ Also, the integrin expression bridging the MSCs to the extracellular matrix proteins COL1A1 and FN may be playing a role, ${ }^{34,38}$ as those proteins were differentially expressed at the lower end of the FN-gradient by the cell population. The superior results from the 10th $\mathrm{mm}$ of the here studied FN density-gradient, were confirmed on homogeneous samples, indicating that paracrine signaling did not contribute to the 


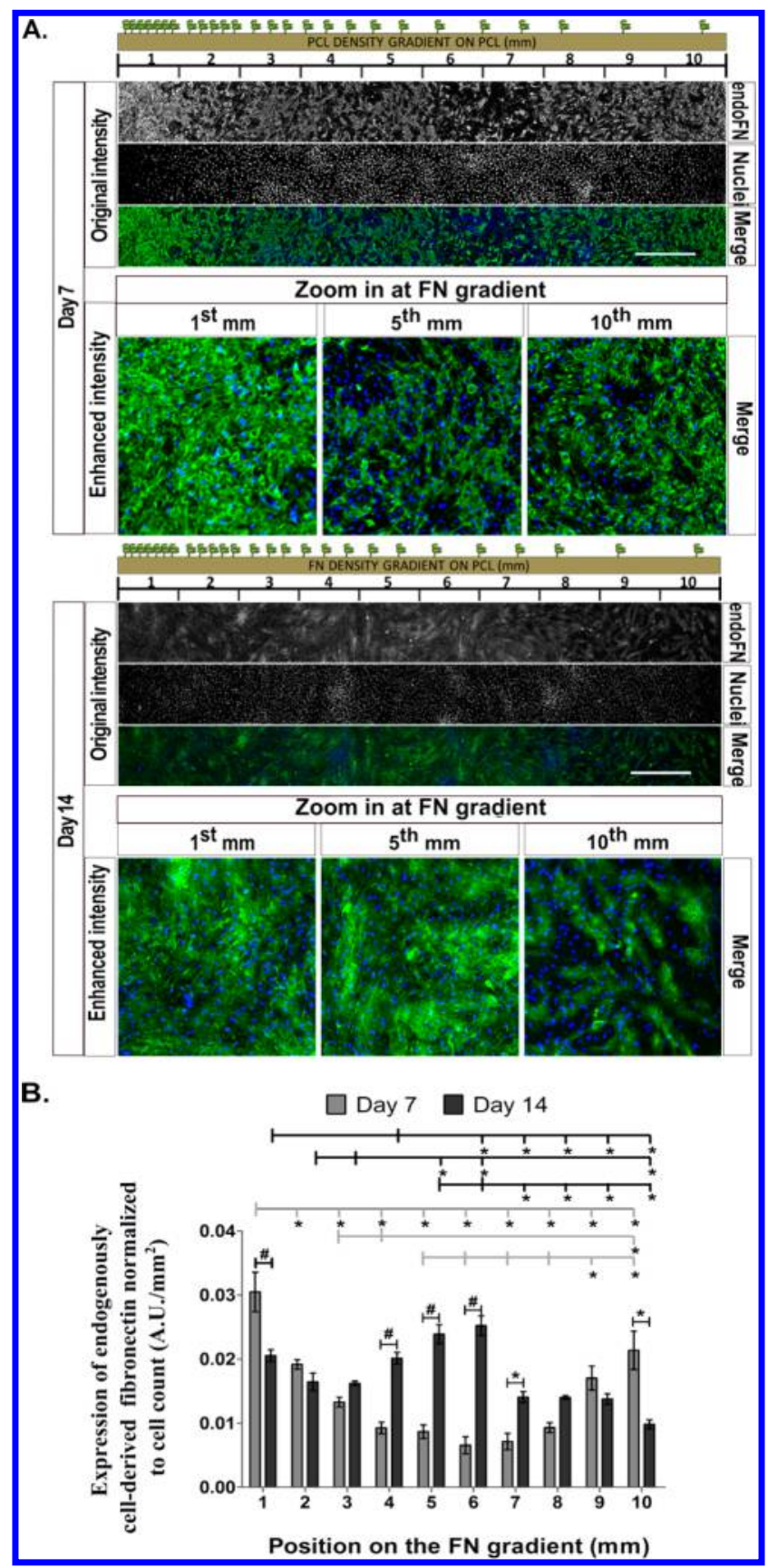

Figure 6. Expression of endogenously cell-derived fibronectin (endoFN) on fibronectin (FN) gradients, at days 7 and 14. (A) Immunodetection of endoFN (scale bar $=1 \mathrm{~mm}$ ). All square images are $1 \times 1 \mathrm{~mm}$. (B) Quantification of endoFN expression, normalized to cell number. The statistical significance of the measurements was $0.001<p(*)<0.05$.

final osteogenic outcome (see Figure S2 in the Supporting Information).

\section{CONCLUSIONS}

The development of a surface-density gradient of $\mathrm{FN}$, on a clinically relevant polymer, as a platform to study the effect of FN density on the osteogenic commitment of hBM-MSCs was successfully accomplished. The analysis revealed that cells cultured in high FN-density areas showed high COL1A1 expression, but medium ALP activity. The intermediate FN densities supported greater cell adhesion and proliferation, which was detrimental for the osteogenic commitment. Specific human FN density at position $10 \mathrm{~mm}$ in the gradient, corresponding to $\sim 48 \mathrm{ng} / \mathrm{cm}^{2}$, consistently supported higher cell spreading and increased expression of COL1A1 and ALP activity, characteristic of osteoblastic differentiation, both in comparison to the remaining positions in the gradient and to the TCPS control We extrapolate that, given proper backfilling passivation, the application of this optimal FN density on materials of similar properties to those of PCL should promote equivalent results as the herein reported. We thus propose a simple and versatile strategy to significantly enhance the biological performance of different synthetic materials intended for bone-related applications involving interaction with human mesenchymal stem cells.

\section{ASSOCIATED CONTENT}

\section{S Supporting Information}

Characterization of the polycaprolatone (PCL)-coating, detailed method of calculation of the adsorbed mass of fibronectin on PCL, cell morphology, and mean area of spreading (Figure S1), (qualitative and quantitative) expression of the osteogenic markers ALP and COL1A1 on homogeneous surface-density control samples (Figure S2). This material is available free of charge via the Internet at http://pubs.acs.org.

\section{AUTHOR INFORMATION}

\section{Corresponding Author}

*E-mail: nuno@dep.uminho.pt. Phone: +351 253510 905. Fax: +351253510909.

\section{Present Addresses}

$\square$ T.O.I. is currently at Stem Cells in Neurological Applications, BioMediTech, University of Tampere, 33520 Tampere, Finland.

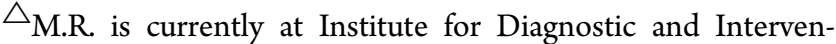
tional Radiology, University Hospital Zürich, CH-8091 Zürich, Switzerland

\section{Author Contributions}

The manuscript was written through contributions of all authors. All authors have given approval to the final version of the manuscript.

\section{Notes}

The authors declare no competing financial interest.

\section{ACKNOWLEDGMENTS}

This work was supported by the Foundation for Science and Technology for the PhD grant of A.B. Faia-Torres (SFRH/BD/ $36476 / 2007)$. We are also grateful to the Swiss National Science foundation (SNSF) grant P300P3_154664 for providing funds to $\mathrm{MC}$.

\section{ABBREVIATIONS}

FN, fibronectin

ALP, alkaline phosphatase

COL1A1, collagen type 1 alpha 1

hBM-MSCs, human bone marrow mesenchymal stem cells

\section{REFERENCES}

(1) Drachuk, I.; Gupta, M. K.; Tsukruk, V. V. Biomimetic Coatings to Control Cellular Function Through Cell Surface Engineering. Adv. Funct. Mater. 2013, 23, 4437-4453.

(2) Martins, A.; Pinho, E. D.; Faria, S.; Pashkuleva, I.; Marques, A. P.; Reis, R. L.; Neves, N. M. Surface Modification of Electrospun 
Polycaprolactone Nanofiber Meshes by Plasma Treatment to Enhance Biological Performance. Small 2009, 5, 1195-1206.

(3) Badylak, S. F.; Freytes, D. O.; Gilbert, T. W. Extracellular Matrix as a Biological Scaffold Material: Structure and Function. Acta Biomater. 2009, 5, 1-13.

(4) Serrano, M. C.; Chung, E. J.; Ameer, G. A. Advances and Applications of Biodegradable Elastomers in Regenerative Medicine. Adv. Funct. Mater. 2010, 2, 192-208.

(5) Boskey, A. L. Bone Composition: Relationship to Bone Fragility and Antiosteoporotic Drug Effects. BoneKey Rep. 2013, 2, 447.

(6) Kadler, K. E.; Hill, A.; Canty-Laird, E. G. Collagen Fibrillogenesis: Fibronectin, Integrins, and Minor Collagens as Organizers and Nucleators. Curr. Opin. Cell Biol. 2008, 20, 495-501.

(7) Erat, M. C.; Sladek, B.; Campbell, I. D.; Vakonakis, I. Structural Analysis of Collagen Type I Interactions with Human Fibronectin Reveals a Cooperative Binding Mode. J. Biol. Chem. 2013, 288, 17441-17450.

(8) Imbert, L.; Aurégan, J.-C.; Pernelle, K.; Hoc, T. Mechanical and Mineral Properties of Osteogenesis Imperfecta Human Bones at the Tissue Level. Bone 2014, 65, 18-24.

(9) To, W. S.; Midwood, K. S. Plasma and Cellular Fibronectin: Distinct and Independent Functions During Tissue Repair. Fibrogenesis Tissue Repair 2011, 4, 21-38.

(10) Caplan, A. I. Why Are MSCs Therapeutic? New Data: New Insight. J. Pathol. 2009, 217, 318-324.

(11) Faia-Torres, A. B.; Guimond-Lischer, S.; Rottmar, M.; Charnley, M.; Goren, T.; Maniura-Weber, K.; Spencer, N.; Reis, R. L.; Neves, N. M. Differential Regulation of Osteogenic Differentiation of Stem Cells on Surface Roughness Gradients. Biomaterials 2014, 35, 9023-9032.

(12) Harris, G. M.; Piroli, M. E.; Jabbarzadeh, E. Deconstructing the Effects of Matrix Elasticity and Geometry in Mesenchymal Stem Cell Lineage Commitment. Adv. Funct. Mater. 2014, 24, 2396-2403.

(13) Caliari, S. R.; Harley, B. A. C. Structural and Biochemical Modification of a Collagen Scaffold to Selectively Enhance MSC Tenogenic, Chondrogenic, and Osteogenic Differentiation. Adv. Healthcare Mater. 2014, 3, 1086-1096.

(14) Zink, C.; Hall, H.; Brunette, D. M.; Spencer, N. D. Orthogonal Nanometer-Micrometer Roughness Gradients Probe Morphological Influences on Cell Behavior. Biomaterials 2012, 33, 8055-8061.

(15) Lamb, B. M.; Nagdas, S.; Yousaf, M. N. Cell Division Orientation on Biospecific Peptide Gradients. ACS Appl. Mater. Interfaces 2014, 6, 11523-11528.

(16) Liu, W.; Lipner, J.; Xie, J.; Manning, C. N.; Thomopoulos, S.; Xia, Y. Nanofiber Scaffolds with Gradients in Mineral Content for Spatial Control of Osteogenesis. ACS Appl. Mater. Interfaces 2014, 6, 2842-2849.

(17) Alves, H.; Munoz-Najar, U.; de Wit, J.; Renard, A. J. S.; Hoeijmakers, J. H. J.; Sedivy, J. M.; van Blitterswijk, C.; de Boer, J. A Link Between the Accumulation of DNA Damage and Loss of MultiPotency of Human Mesenchymal Stromal Cells. J. Cell. Mol. Med. 2010, 14, 2729-2738.

(18) Ghaemi, S. R.; Harding, F.; Delalat, B.; Vasani, R.; Voelcker, N. $\mathrm{H}$. Surface Engineering for Long-Term Culturing of Mesenchymal Stem Cell Microarrays. Biomacromolecules 2013, 14, 2675-2683.

(19) Feller, L. M.; Cerritelli, S.; Textor, M.; Hubbell, J. A.; Tosatti, S. G. P. Influence of Poly(Propylene Sulfide-Block-Ethylene Glycol) Diand Triblock Copolymer Architecture on the Formation of Molecular Adlayers on Gold Surfaces and Their Effect on Protein Resistance: A Candidate for Surface Modification in Biosensor Research. Macromolecules 2005, 38, 10503-10510.

(20) Engvall, E.; Ruoslahti, E. Binding of Soluble Form of Fibroblast Surface Protein, Fibronectin, to Collagen. Int. J. Cancer 1997, 20, 1-5.

(21) Heuberger, M.; Balmer, T. E. The Transmission Interferometric Adsorption Sensor. J. Phys. Appl. Phys. 2007, 40, 7245-7254.

(22) Schindelin, J.; Arganda-Carreras, I.; Frise, E.; Kaynig, V.; Longair, M.; Pietzsch, T.; Preibisch, S.; Rueden, C.; Saalfeld, S.; Schmid, B.; Tinevez, J.-Y.; White, D. J.; Hartenstein, V.; Eliceiri, K.; Tomancak, P.; Cardona, A. Fiji: an Open-Source Platform for Biological-Image Analysis. Nat. Methods 2012, 9, 676-682.
(23) Studer, D.; Lischer, S.; Jochum, W.; Ehrbar, M.; Zenobi-Wong, M.; Maniura-Weber, K. Ribosomal Protein 113a as a Reference Gene for Human Bone Marrow-Derived Mesenchymal Stromal Cells During Expansion, Adipo-, Chondro-, and Osteogenesis. Tissue Eng., Part C 2012, 18, 761-771.

(24) Feijter, J. A. D.; Benjamins, J.; Veer, F. A. Ellipsometry as a Tool to Study the Adsorption Behavior of Synthetic and Biopolymers at the Air-Water Interface. Biopolymers 1978, 17, 1759-1772.

(25) Hynes, R. O. The Extracellular Matrix: Not Just Pretty Fibrils. Science 2009, 326, 1216-1219.

(26) Lu, P.; Takai, K.; Weaver, V. M.; Werb, Z. Extracellular Matrix Degradation and Remodeling in Development and Disease. Cold Spring Harb. Perspect. Biol. 2011, 3, a005058-74.

(27) Era, S.; Sogami, M.; Uyesaka, N.; Kato, K.; Murakami, M.; Matsushima, S.; Kinosada, Y. Comparative Intermolecular CrossRelaxation Studies of Human Hemoglobin in Red Blood Cells and Bovine Serum Albumin in Solution. NMR Biomed. 2011, 24, 483-491.

(28) Latour, R. A. Biomaterials: Protein-Surface Interactions. The Encyclopedia of Biomaterials and Bioengineering, 2nd ed.; Informa Healthcare: Zug, Switzerland, 2008.

(29) Ramanathan, A.; Karuri, N. Fibronectin Alters the Rate of Formation and Structure of the Fibrin Matrix. Biochem. Biophys. Res. Commun. 2014, 433, 395-399.

(30) Cavalcanti-Adam, E. A.; Volberg, T.; Micoulet, A.; Kessler, H.; Geiger, B.; Spatz, J. P. Cell Spreading and Focal Adhesion Dynamics Are Regulated by Spacing of Integrin Ligands. Biophys. J. 2007, 92, 2964-2974.

(31) Bradshaw, M. J.; Smith, M. L. Multiscale Relationships Between Fibronectin Structure and Functional Properties. Acta Biomater. 2014, $10,1524-1531$.

(32) Zhou, Y.; Guan, X.; Zhu, Z.; Gao, S.; Zhang, C.; Li, C.; Hou, W.; $\mathrm{Yu}, \mathrm{H}$. Osteogenic Differentiation of Bone Marrow-Derived Mesenchymal Stromal Cells on Bone-Derived Scaffolds: Effect of Microvibration and Role of ERK1/2 Activation. Eur. Cell. Mater. 2011, $22,12-25$.

(33) McBeath, R.; Pirone, D. M.; Nelson, C. M.; Bhadriraju, K.; Chen, C. S. Cell Shape, Cytoskeletal Tension, and RhoA Regulate Stem Cell Lineage Commitment. Dev. Cell 2004, 6, 483-495.

(34) Schwab, E. H.; Halbig, M.; Glenske, K.; Wagner, A.-S.; Wenisch, S.; Cavalcanti-Adam, E. Distinct Effects of RGD-Glycoproteins on Integrin-Mediated Adhesion and Osteogenic Differentiation of Human Mesenchymal Stem Cells. Int. J. Med. Sci. 2013, 10, 1846-1859.

(35) Linsley, C.; Wu, B.; Tawil, B. The Effect of Fibrinogen, Collagen Type I, and Fibronectin on Mesenchymal Stem Cell Growth and Differentiation Into Osteoblasts. Tissue Eng. Pt. A 2013, 19, 14161423.

(36) Sottile, J.; Hocking, D. C. Fibronectin Polymerization Regulates the Composition and Stability of Extracellular Matrix Fibrils and CellMatrix Adhesions. Mol. Biol. Cell 2002, 13, 3546-3559.

(37) Weiss, R. E.; Reddi, A. H. Appearance of Fibronectin During the Differentiation of Cartilage, Bone, and Bone marrow. J. Cell Biol. 1981, $88,630-636$.

(38) Li, B.; Moshfegh, C.; Lin, Z.; Albuschies, J.; Vogel, V. Mesenchymal Stem Cells Exploit Extracellular Matrix as Mechanotransducer. Sci. Rep. 2013, 3, 2425-2433.

(39) Bentmann, A.; Kawelke, N.; Moss, D.; Zentgraf, H.; Bala, Y.; Berger, I.; Gasser, J. A.; Nakchbandi, I. A. Circulating Fibronectin Affects Bone Matrix, Whereas Osteoblast Fibronectin Modulates Osteoblast Function. J. Bone Miner. Res. 2010, 25, 706.

(40) Mathieu, P. S.; Loboa, E. G. Cytoskeletal and Focal Adhesion Influences on Mesenchymal Stem Cell Shape, Mechanical Properties, and Differentiation Down Osteogenic, Adipogenic, and Chondrogenic Pathways. Tissue Eng., Part B 2012, 18, 434. 